\title{
Modelagem e Controle de um Sistema TITO de Processo Industrial mediante o Protocolo OPC e o FOSS Scilab
}

\author{
Accacio Ferreira dos Santos Neto ${ }^{1}$ \\ Centro Federal de Educação Tecnológica de Minas Gerais, CEFET-MG \\ Departamento de Eletroeletrônica, Campus Leopoldina, MG \\ Francisco José Gomes ${ }^{2}$ \\ Universidade Federal de Juiz de Fora, UFJF \\ Faculdade de Engenharia, Departamento de Energia Elétrica, Juiz de Fora, MG
}

\begin{abstract}
Resumo. Este trabalho apresenta o projeto e a montagem de um módulo didático para modelagem e controle de um sistema multivariável do tipo TITO, para dois processos complexos, utilizando o Protocolo OPC e FOSS Scilab, que permitiram o desenvolvimento de um sistema de grande utilidade tanto para treinamento de operadores industriais quanto para a pesquisa acadêmica.
\end{abstract}

Palavras-chave. Modelagem, Controle Automático, Sistema TITO, Protocolo OPC, Scilab

\section{Introdução}

A disponibilidade da instrumentação eletrônica tem motivado e impulsionado a expansão da automação das plantas industriais, da indústria de processos e o crescimento do setor [5]. Nota-se grande diversidade de softwares e dispositivos resultantes deste desenvolvimento, o que permite suprir muitas das necessidades da sociedade. Por outro lado, é muito comum notar, ao se adentrar em uma indústria e observar uma planta de processo industrial, a grande variedade de softwares e hardwares existentes (Schneider, Weg, ABB, entre outros fabricantes), fato este que, em certas situações, proporciona dificuldades de comunicação entre os hardwares de fabricantes diferentes ("interoperabilidade"), custos adicionais no que se refere à necessidade de desenvolvimento de "drivers" de comunicação entre softwares ou mesmo a dependência a determinados fabricantes.

Observa-se, neste contexto, uma tendência para o desenvolvimento de plataformas computacionais que proporcionem avanços nesta área e que impactam diretamente vários setores de inovação tecnológica como, por exemplo, os sistemas "Free Open Source Software - FOSS". A pesquisa de sistemas via FOSS insere-se nestas diretrizes e tem sido objeto de investimentos de diversas agências de fomento internacionais, artigos

accacio@leopoldina.cefetmg.br

chico.gomes@ufjf.edu.br 
em periódicos e sessões de congressos internacionais [1, 3, 9, 11, 14].

Nesta direção, foram estabelecidas a motivação e o objetivo do presente trabalho ao propor procedimentos que auxiliem a contornar obstáculos de projeto e implementação de técnicas de controladores para processos industriais buscando facilidade, avaliação e aplicabilidade em sistemas dinâmicos, baseados em FOSS, com benefícios para o desenvolvimento de pesquisa acadêmica, treinamento de operadores e linhas de inovação. Em essência, a concepção principal deste trabalho tem como base a utilização de padrões abertos de protocolos de comunicação, que vêm sendo difundidos nos ambientes industriais e chãos-de-fábrica, mais especificamente, o protocolo "OLE for Process Control - OPC" [16]. Utilizando as regras deste protocolo foi possível o desenvolvimento de um módulo didático, com um supervisório inteiramente baseado no FOSS Scilab [14], possibilitando seu controle e gerenciamento, e englobando duas malhas típicas de processos industriais, interativas, com características singulares: uma malha de controle de nível, com característica integrativa, e uma malha de controle de temperatura, com elevado atraso de transporte.

O trabalho está estruturado como segue: a seção 2 descreve a metodologia utilizada no projeto, abordando o Protocolo OPC, o FOSS Scilab e a descrição do módulo físico; a seção 3 apresenta e discute os resultados experimentais obtido e as conclusões finais são abordadas na seção 4 .

\section{Metodologia}

A metodologia utilizada baseou-se na associação do FOSS Scilab e do Protocolo de comunicação OPC. Neste contexto, o protocolo de comunicação é a base de toda a concepção, possibilitando o controle do sistema físico através de algoritmos implementados e operados a partir do supervisório desenvolvido em Scilab. É possível observar-se, de forma esquemática, na figura 1, a relação do software Scilab, instalado em um PC, e o módulo físico desenvolvido, mediados pelo protocolo OPC. O Controlador Lógico Programável - CLP, trabalha como o equipamento que realiza a interface entre supervisório desenvolvido em Scilab, no PC, e o módulo físico.

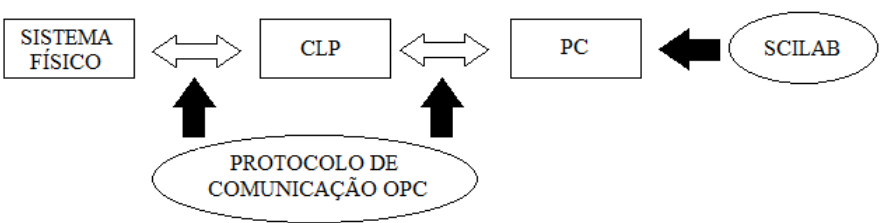

Figura 1: Esquemático da metodologia proposta

\subsection{Protocolo OPC}

O protocolo OPC é um protocolo de comunicação de dados, cuja sigla é o acrônimo de "OLE for Process Control" - OPC, que consiste na versão OLE - "Object Linking and Embedding" - para a indústria de automação $[12,16]$. Constitui-se de um conjunto comum de interfaces, métodos e propriedades de comunicação, agregados dentro de uma especificação padronizada e aberta ao acesso público [7], que surgiu em 1996 em meio a dois aspectos principais: a existência de soluções proprietárias dos equipamentos de fornecedores diversificados e redes de campo a eles associadas. Vale destacar, nesta tecnologia, o fato de oferecer um mecanismo padrão para comunicação e 
troca de dados entre servidores e clientes de fabricantes distintos, suportando aplicação em redes de comunicação de procedimentos [16]. Para um reforço da compreensão sobre o protocolo, é válido lembrar que a tecnologia OLE da Microsoft, surgiu em função da necessidade de desenvolver "drivers" específicos para cada fabricante de impressora diferente. Deste modo, de maneira análoga à dificuldade de interoperabilidade na indústria, a padronização eliminou a necessidade de softwares auxiliares para cada impressora [12].

\subsection{FOSS Scilab}

Dentre a variedade de softwares disponibilizada pelo mercado, os que remetem à computação numérica adquirem, de forma crescente, grande importância, principalmente pelas vantagens proporcionadas por sua flexibilidade e pelo uso das simulações, que reduzem custos e tempo. Por outro lado, muitos destes softwares não são de baixo custo, o que pode se tornar um empecilho à sua utilização. Registra-se, neste contexto, o aparecimento de softwares livres como o Scilab e o Octave [7], que surgiram na busca de uma solução FOSS para o Matlab, cuja licença não é livre, nem barata. Neste cenário, o Scilab [6] surge como a solução para várias aplicações, inclusive para o controle de processos industriais $[6,13,14]$, pois permite o trabalho com o protocolo OPC.

Dentre suas funcionalidades, como o acesso avançado a estruturas de dados e centenas de funções matemáticas onde destacam-se, dentre outras [7, 14]: operações matemáticas e análise de dados para aplicações de engenharia e científicas; visualização de funções gráficas, bem como registro e exportação de arquivos; desenvolvimento de aplicações através do fornecimento de APIs ("Application Programming Interface") que permitem a criação de módulos próprios, como interfaces interativas e gerenciamento de trocas de dados com ferramentas externas. No desenvolvimento deste trabalho utilizou-se, basicamente: gerenciamento de trocas de informações com aplicações externas (Protocolo OPC), utilização de APIs, operações matemáticas com bibliotecas de funções fornecidas (desenvolvimentos de algoritmos de controle) e ferramentas de visualização gráfica. Observa-se, na figura 2, a real interação do Scilab com o restante do sistema:
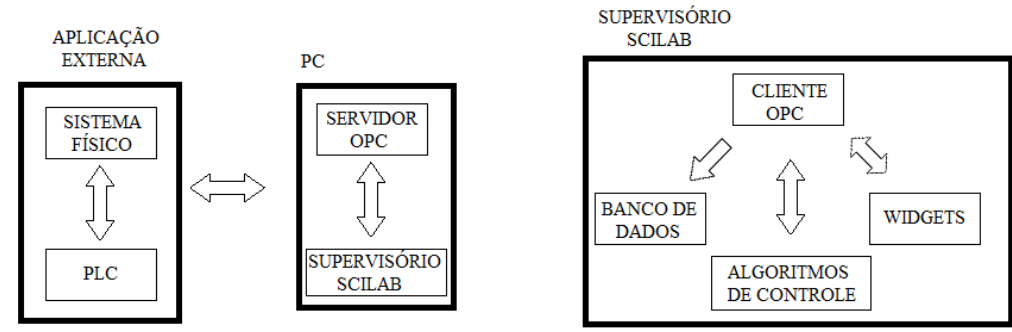

Figura 2: Relação do Scilab com o Sistema Físico

\subsection{Módulo Físico (Sistema TITO)}

Como abordado no início do trabalho, efetuou-se a construção de um módulo laboratorial que possibilitasse a implementação da proposta, envolvendo a utilização de técnicas de modelagem e controle de um sistema com dinâmicas industriais típicas. Desenvolveu-se, assim, um sistema com dinâmicas comuns no ambiente industrial, com especificidades que as distinguisse das características usuais dos processos convencionais [4]: um sistema do tipo "Two-Input Two-Output - TITO", com uma malha de nível, de 
características integrativas, e uma malha de temperatura, com elevado atraso de transporte, interativas. Na figura 3 é possível observar o módulo físico desenvolvido (lado esquerdo da figura) e seu esquemático no lado direito, e seu interfaceamento com o computador. Maiores informações e detalhamentos operacionais podem ser encontrados em [10].
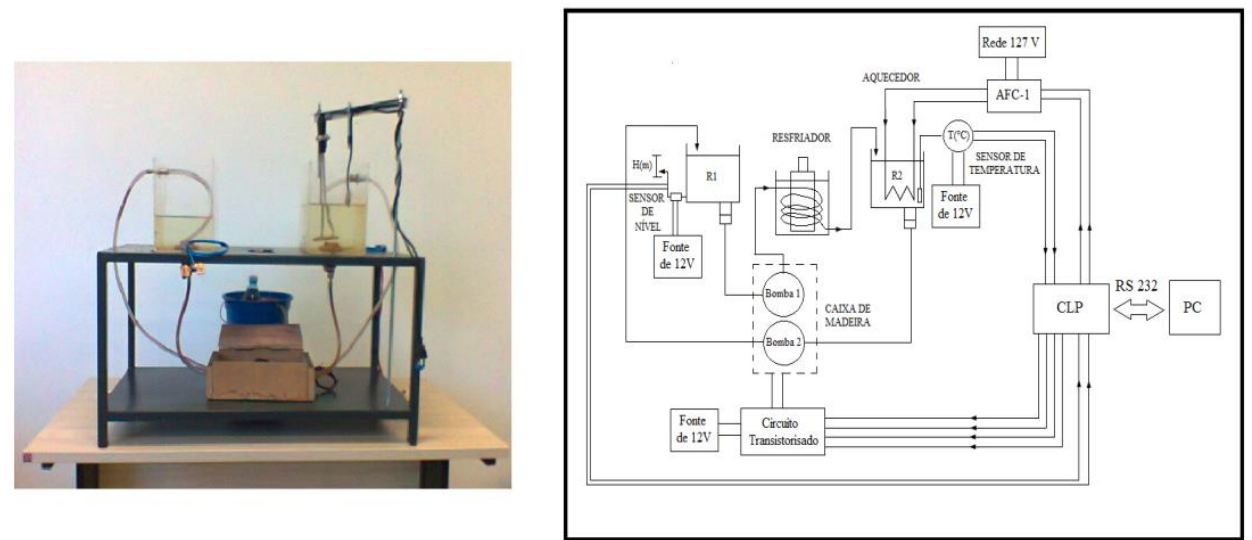

Figura 3. Módulo Físico e esquemático de sua comunicação com o PC

\section{Resultados Experimentais}

Para ilustrar a operacionalidade da proposta serão apresentados alguns resultados, dentre as quais a técnica denominada Controle Seletor, também conhecida como "Override", muito usual na indústria [15]. Para aplicação dessa técnica de controle é necessário definir, inicialmente, as variáveis restritivas e, posteriormente, a sintonia dos controladores responsáveis por seu controle [15], como apresentado na figura 4:

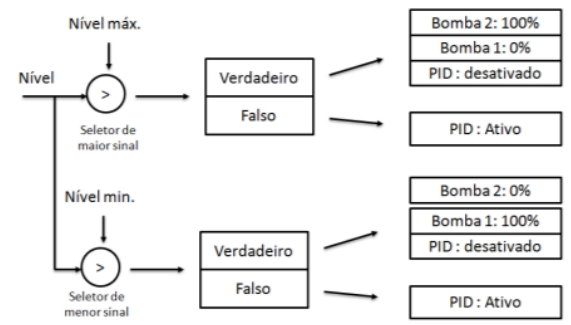

Figura 4: Estrutura do Controle Seletor

Observa-se que o controle da temperatura (controlador PID) mantêm-se ativo enquanto os limites restritivos não são extrapolados, mas o controle das restrições ("on-off") trabalham em situação contrária. A sintonia do controlador principal segue o tradicional procedimento de um sistema do tipo SISO [4]. Aplicando um degrau na variável principal observa-se (figura 5): 

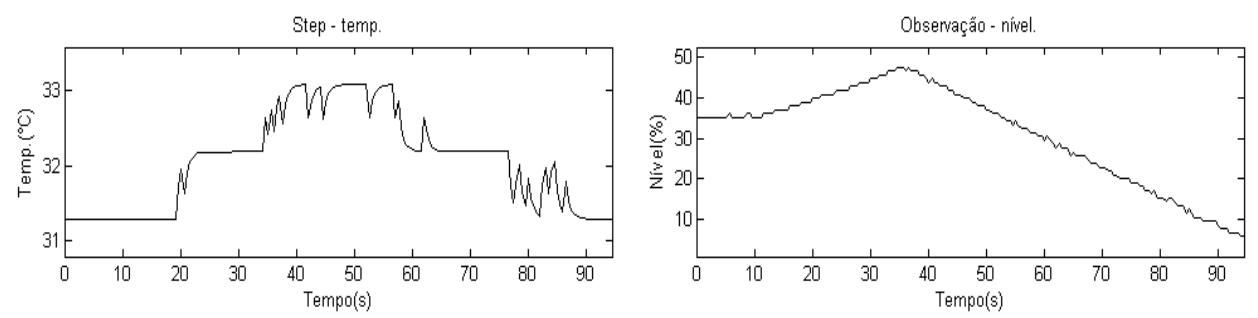

Figura 5. Reposta ao Degrau na Temperatura e Nível do Reservatório

Utilizando os resultados do teste degrau do sistema determinou-se o modelo do sistema físico para definir a sintonia dos controladores inseridos na malha de controle. A seleção dos métodos de sintonia utilizou os métodos clássicos, apresentadas, para caráter comparativo, na Tabela 1.

Tabela 1: Sintonia do PID - Controle Seletor

\begin{tabular}{|c|c|c|c|c|c|c|}
\hline PID - Escravo & \multicolumn{3}{|c|}{ Degrau Positivo } & \multicolumn{3}{|c|}{ Degrau Negativo } \\
\hline Controlador PID & $K p$ & $T i$ & $T d$ & $\mathrm{Kp}$ & $\mathrm{Ti}$ & $\mathrm{Td}$ \\
\hline Preditor de Smith + IMC & 38,17 & 9,79 & - & 49,37 & 18,33 & - \\
\hline Skogestad & 19,08 & 9,79 & - & 24,69 & 18,33 & - \\
\hline
\end{tabular}

Para determinar o comportamento da topologia de controle utilizada, testou-se o sistema físico utilizando-se um supervisório, desenvolvido em inteiramente em Scilab (figura 6), com mudança de referência positiva da temperatura inicial (variação de $\Delta=2^{\circ} \mathrm{C}$ entre $0 \mathrm{e}$ 100 s) e negativa (variação de $\Delta=-2^{\circ} \mathrm{C}$ entre 100 e 200 s); as variáveis de restrições foram ajustadas em limite inferior do nível (40\%) e o limite superior do nível (70\%). O ensaio realizado conduziu aos resultados da figura 6 :
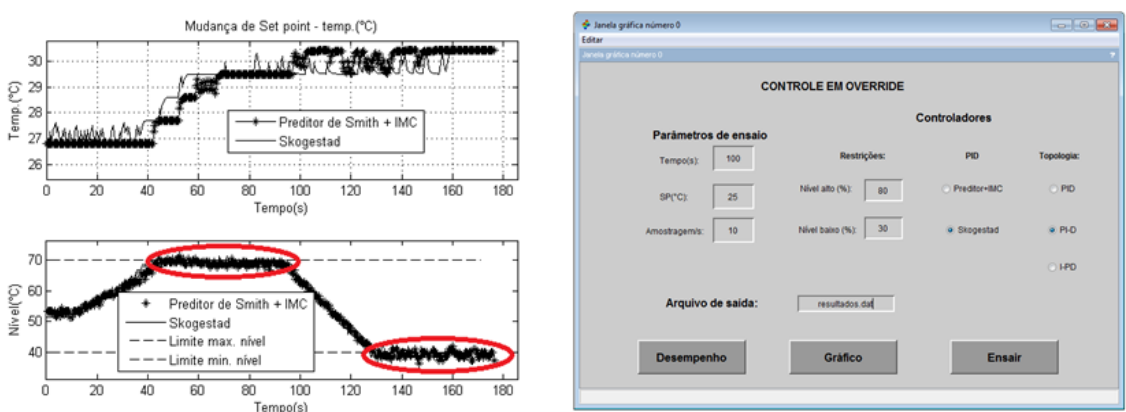

Figura 6: Resultados do Controle Seletor e do Supervisório

Os resultados evidenciam as dinâmicas resultantes das mudanças de referências, observadas principalmente através do comportamento do nível. Nota-se a interferência das restrições que modificam o resultado do controle apresentados na figura em asterisco ("*"). Quanto ao degrau negativo, vale observar o nível a partir dos 100 segundos de ensaio, pois no prosseguimento do processo o controle não permite a perda de massa do reservatório para arrefecer o outro reservatório. Enfim, compreende-se o comportamento do sistema e sua operação de acordo com a proposta da técnica de controle selecionada, dentro das limitações da planta física.

Ressalta-se, também, o supervisório desenvolvido em Scilab, ilustrado ao lado dos 
resultados do ensaio, que apresenta uma interface totalmente "amigável" além de permitir o controle do sistema de um usuário através de Widgets (botões, lacunas), gráficos, avaliação de desempenho e banco de dados (arquivo de saída).

\section{Conclusões}

Em face dos resultados apresentados é possível compreender a aplicabilidade da metodologia proposta que, através da associação de ferramentas FOSS (Scilab e Protocolo OPC), proporcionou o desenvolvimento de um supervisório capaz de controlar satisfatoriamente um sistema físico com grande similaridade aos sistemas encontrados na indústria. Observa-se, também, que a utilização do software Scilab permitiu e permite o desenvolvimento de sistemas supervisórios totalmente flexíveis para variadas aplicações, permitindo a modelagem e o controle de sistemas de modo satisfatório. Quanto ao Protocolo OPC apreende-se, também, sua potencialidade e reafirmação de sua concepção inicial, que desempenha um papel importantíssimo na metodologia proposta e proporciona maiores potencialidades para sua aplicação.

Deste modo, através destes estudos iniciais, é possível vislumbrar novas perspectivas para o desenvolvimento de sistemas capazes de modelar e controlar processos industriais. Enfim, buscando facilidade, flexibilidade, custo benefício através de ferramentas de código aberto, que também podem proporcionar desenvolvimento de pesquisas acadêmicas, treinamentos de operadores e abertura de linhas de inovação.

\section{Agradecimentos}

Agradecemos à FAPEMIG e à instituição CEFET-MG.

\section{Referências}

[1] K. E. Arzen, A. Blomdell and B. Wittenmark, Laboratories and Real-Time Computing: Integrating Experiments into Control Courses, IEEE Control Systems Magazine, vol. 25, 30-34, (2005), DOI: 10.1109/MCS.2005.1388797.

[2] D. S. Barroso, J. A. Quintino e F. J. Gomes, Protocolo OPC somado a sistemas de controles industriais desenvolvidos com Java e Scilab, Proceeding Series of the Brazilian Society of Computational and Applied Mathematics, vol. 1, (2013), DOI: 10.5540/03.2013.001.01.0111.

[3] R. Bucher and S. Balemi, Rapid Control Prototyping using Linux Real-Time. Conference in Control Education (2003).

[4] M. C. M. M. Campos e H. C. G Teixeira, Controle Típico de Equipamentos e Processos Industriais, Edgard Blucher, (2007).

[5] R. M. V. Gutierrez e S. S. K. Pan, Complexo Eletrônico: Automação do Controle Industrial. BNDES Setorial, n. 28, 189-232 (2008).

[6] L. Hladowski, B. Cichy, K. Galkowski, B. Sulikowski and E. Rogers, SCILAB 
compatible software for analysis and control of repetitive processes, IEEE Conference on Computer Aided Control Systems Design, 3024-3029, (2006).

[7] W. Liao, N. Dong and T. Fan, The application of Scilab/Scicos in the lecture of automatic control theory, Open-source Software for Scientific Computation, IEEE International Workshop on, 85-87, (2009), DOI: 10.1109/OSSC.2009.5416865.

[8] A. B. Lugli, e M. M. D. Santos, Sistemas Fieldbus para Automação Industrial: DeviceNet, CANopen, SDS e Ethernet, Érica Ltda, (2009).

[9] C. Meza, J. A. Andrade-Romero and S. Balemi, Free Open Source Software in Control Engineering Education: A Case Study in the Analysis and Control Design of a Rotary Inverted Pendulum. IEEE Conference on Emerging Technologies \& Factory Automation, 1-8, (2009), DOI:10.1109/ETFA.2009.5347162.

[10] A. F. S. Neto, Aplicação do Protocolo Aberto OPC e do FOSS Scilab no Desenvolvimento de um Módulo Laboratorial para Controle de Processos Industriais, Dissertação de Mestrado em Modelagem Computacional, UFJF, (2013).

[11] P. S. M. Pires and D. A. Rogers, Free/open source software: An alternative for engineering students, Frontiers in Education, vol.1, (2002), DOI: 10.1109/FIE.2002.1157982.

[12] A. P. Puda, Padronização da comunicação através da tecnologia OPC, Soft-Brasil Automação, (2008).

[13] E. M. Silva e J. P. V. S. Cunha, Scilab, scicos e rltool: Softwares livres no ensino de engenharia elétrica, Anais do Congresso Brasileiro de Automática, 1620-1625, (2006).

[14] P. Tona, Teaching Process Control with Scilab and Scicos. American Control Conference, 280-285, (2006), DOI: 10.1109/ACC.2006.1655368.

[15] H. L. Wade, Basic and Advanced Regulatory Control: System Design and Application, ISA, (2004).

[16] L. Zheng and H. Nakagawa, OPC (OLE for process control) specification and its developments, Proceedings of the 41st SICE Annual Conference, vol. 2, 917-920, (2002), DOI: 10.1109/SICE.2002.1195286. 